University of East London Institutional Repository: http://roar.uel.ac.uk

This paper is made available online in accordance with publisher policies. Please scroll down to view the document itself. Please refer to the repository record for this item and our policy information available from the repository home page for further information.

To see the final version of this paper please visit the publisher's website. Access to the published version may require a subscription.

Author(s): Rustin, Michael

Article Title: Looking in the Right Place: Complexity Theory, Psychoanalysis and Infant Observation.

Year of publication: 2002

Citation: Rustin, M. (2002) 'Looking in the Right Place: Complexity Theory, Psychoanalysis and Infant Observation', Infant Observation 5 (1) 122 -144

Link to published version: http://dx.doi.org/10.1080/13698030208401653

DOI: $10.1080 / 13698030208401653$

Publisher statement: http://www.tandf.co.uk/journals/copyright.asp

Information on how to cite items within roar@uel:

http://www.uel.ac.uk/roar/openaccess.htm\#Citing 
Text of article published in The International Journal of Infant Observation. Vol. 5 No, 1 (2002), and in Andrew Briggs (ed). Surviving Space: Papers on Infant

Observation. Karnac/Tavistock Clinic Book Series (2002).

\title{
Looking in the Right Place: Complexity Theory, Psychoanalysis and Infant Observation. ${ }^{1}$
}

\author{
Michael Rustin
}

After over fifty years of the practice of Esther Bick's model of infant observation, there surely cannot be too much doubt about what is supposed to be observed in this setting. Esther Bick (1964) herself described the purposes of introducing infant observation into the curriculum of child psychotherapists as to 'help students to conceive vividly the infantile experience of their child patients.' She refers also to 'the student's understanding of the child's non-verbal behaviour,' and to the student's 'unique opportunity to observe the development of an infant more or less from birth, and in his home setting and in his relation to his immediate family, and thus to find out for himself how these relations emerge.'

This is, in descriptive terms, clear enough, and has been a good enough guide to the educational purposes of infant observation. But when one thinks of infant observation as a resource for generating new ideas and understandings in psychoanalysis - that is for research- it says rather little. For this purpose, a more theoretical discussion of the kinds of data and experience that infant observation can provide, and how these relate to the development of psychoanalytic theory, is necessary. I have explored these issues in two previous papers, (Rustin 1989 and 1997), and in this one, drawing on the writings of both Bick and Bion, I add some additional 
dimensions. I will draw attention to some interesting parallels between the modelling and mapping devices developed in recent work in Complexity Theory and Chaos Theory (originating in mathematics, but now with broadening applications to empirical sciences), and psychoanalytic method, and then suggest how these are relevant to infant observation too. I shall be suggesting that Bion, and in more implicit way Bick, anticipated the ways of thinking of Complexity Theory, from their very different psychoanalytic starting point, by about twenty years.

There is an unavoidably abstract and meta-theoretical aspect to these research questions, which involve the definition of our underlying objects of study. What kinds of meaning are we looking for in clinical and observational studies? Are they the kinds of regularities which are summarised in scientific laws, defining relations of cause and effect? Can they be generalised between instances? Can empirical evidence be found for such correlations as we identify? Or are the links between phenomena that we see merely relations of logical coherence and consistency, - what we think of as the subjective meaning of an action or a state of mind, when we understand how states of feeling, desire and belief are connected to one another? For example, we may understand an envious personality as one which is dominated by a pervasive (perhaps largely unconscious) disposition or feeling, which gives meaning and coherence to various particular beliefs and actions of the subject.

Psychoanalytic explanation tends to waver between these different poles of cause and meaning. It needs the dimension of subjective and unconscious meaning to give any sense at all to its work, but also finds it difficult to do without some idea of causal connection or law-like relationship, to the discovery and accumulation of which of course Freud was in particular dedicated. Different research programmes in psychoanalysis and its adjacent fields are shaped by these different polarities. The advocates of 'empirical research' in psychoanalysis seek to make psychoanalysis more compatible with 'scientific' methods in which valid and reliable measures of

\footnotetext{
${ }^{1}$ This is the complete version of a paper presented at the Conference 'Frontiers of Practice 2: The New Dialogue between Attachment Theory and British Object Relations', Bellevue WA.
} 
causal relations are fundamental. Those on the contrary committed to clinical methods tend to stay closer to the dimensions of subjective meaning, and to the individual case-studies in which these are most vividly manifested. The methodological difficulty for the latter is in finding systematic ways of generalising from individual instances. The methodological difficulty for the former is to avoid such flattening out of individual differences, and of the sheer complexity of psychic phenomena, in their search for verifiable laws, that they move far away from a psychoanalytic understanding. ${ }^{2}$

This polar opposition between dimensions of causality and meaning has long divided studies in the sciences, which once seemed to be unambiguously concerned with causality, from those in the humanities, which seemed largely concerned with meanings. The 'human sciences' have sat uncomfortably in the middle of the division, some of them (such as psychology and economics) aiming to be as science-like as possible, whilst others, more preoccupied with dimensions of culture, such as anthropology and some branches of sociology, have given more weight to description - 'thick description', as Clifford Geertz (1973) called it - over causal explanation, as the only way truthfully to render the complexity and contingency of human experience. Psychoanalysis has largely located itself on the subjective and meaningful end of this continuum. Kant's philosophical distinctions between the causallydetermined world of nature, and the human domain of freedom and selfgenerated order have long provided an underlying metaphysical framing for this polarity of approaches. ${ }^{3}$

The persistence and intractability of this opposition might suggest however that the problems have been hitherto framed in a misleading way. This seems especially suggested by the fact that in the human sciences both

\footnotetext{
${ }^{2}$ Reflections on the relevance of complexity theory to the debate on evidence-based psychotherapy are in Robinson (2002). On the broader issues of evidence, see Rustin (2001b).

3 The development of complexity theory, and its implications for psychoanalysis, to some degree however match Kant's own resolution of this antithesis so far as human understanding is concerned, in his own account of the order-creating capacities of the human mind, in The Critique of Judgement. The post-Kleinian emphasis on the functions of mind and on the aesthetic sense as a manifestation of this has affinities with the argument of the Critique of
} 
sides in this argument have often sought to overcome the limitations of their partial position by incorporating some of the insights of their antagonists.

'Causal' approaches try to introduce a dimension of meaning, for example by defining 'meanings' and intentions as a particular kind of cause. Approaches focusing on meaning nevertheless identify developmental patterns, and selfmaintaining 'systems' or kinds of order, which seem to advance weak but unmistakable assertions of causal connection. Perhaps there might be another way of formulating the issues that can go beyond such pragmatic compromises between approaches, into a more adequate formulation of what 'binds' human actions together.

\section{Complexity Theory}

I suggest we might find the more adequate formulations we need in the field of ideas known as 'Complexity Theory' and 'Chaos Theory', 4 (Ruelle 1991, Gleick 1998, Prigogine and Stengers 1984, Stewart 1990, Prigogine 1996, Gell-Mann 1994, Kauffman 1995) which is just beginning to have a substantial impact on the human sciences (Byrne 1998, Thrift 1999, Eve, Horsfall and Lee 1997), and on the ways in which scientific explanations are framed. ${ }^{5}$

In the last twenty or so years, a major field of inquiry has developed around the significance in the natural, biological, and social sciences, of complex self-organising systems. The properties of these systems are not explicable or predictable by reference to models of linear causation. Of particular

Judgement, as both Likierman (1989) and Rustin (1991c) have pointed out in their discussions of psychoanalytic aesthetics.

${ }^{4}$ Chaos theory, developed initially by mathematicians, and physical scientists, sought to investigate unexpected properties of order in apparently chaotic or random environments. 'Complexity theory' emerged subsequently from this, among scientists interested mainly in self-organising biological systems. A literal and metaphorical boundary between these two overlapping frames of reference is the concept of 'the edge of chaos', One hypothesis, developed through computer modelling of various evolutionary processes, is that the 'edge of chaos' provides the optimal environment for development. 'It is as though a position in the ordered regime near the transition to chaos affords the best mixture of stability and flexibility.' (Kauffman 1995, p. 91). Complexity theory became something of a social movement, its methodological holism, and its idea that order could be discerned in turbulent environments, giving a meta-theoretical backing to the idea of 'sustainability'. For a brief account of this social dimension, see Waldrop (1992) chapter 9. 
interest within this paradigm are changes from one ordered systemic state to another, and the role of contingencies in bringing such changes about. They involve time-irreversible processes, and are not neutral with respect to time as have been the models of both classical and quantum physics. They have the attribute that small changes in initial conditions can generate very large divergences in systemic outcomes (the famous 'butterfly effect'; in which the fluttering of the wings of a butterfly in the Amazon rain forest could in theory have major effects on a weather system in the United States). These systematic organisations show a tendency for 'bifurcation', such that from a single starting point alternative structured outcomes are possible. Finally, there is a tendency for coherence and cohesion within such systems, which is explained by what are described in an extraordinarily resonant metaphor as 'strange attractors'. 6 'Strange attractors' describe the forces for cohesion and order in multi-dimensional systems whose states cannot be explained as the outcome of linear causal principles. One analogy used in this literature to make sense of 'attractors' is that of neighbouring river basins, into one or other of which all the drainage of a locality must flow.

This paradigm identifies and explores a new kind of order, intermediate between the deterministic order of classical physics, and the spheres of the apparently random and unpredictable. 'Chaos', as defined within 'chaos theory', is not chaos or randomness as this is understood in commonsense terms, but has its own different ordering principles, marked by major transitions, bifurcations of development, multi-dimensional causality, 'emergent properties'; which are the outcome of interactions between entities within systems, and of time-irreversible evolutionary patterns. The assumptions of constancy, determinism, and equilibrium, which underlay previously conventional scientific paradigms, are replaced by the idea of evolution, partial uncertainty, and disequilibrium. The earlier assumptions (e.g. of classical physics) remain valid within the assumption of 'closed systems'. However, it is argued that the existence of closed systems cannot

\footnotetext{
${ }^{5}$ A useful overview of this field is Mark C. Taylor (2001) The Moment of Complexity: Emerging Network Culture.
} 
be presupposed. (It is surely certain that human personality and social systems never have the attributes of closed systems.) Quantum theory also suggests that at micro-levels a principle of indeterminacy holds (Heisenberg's 'uncertainty principle' being one famous version of this). This suggests that the actual world which we know through our 'coarse grained' modelling is only one possible world of the many that might have eventuated from the properties of nature. This is a fortiori true of biological evolution, and of course of the evolution of culture and society, where there is no reason to presuppose a law-like determination of one predestined present or future. ${ }^{7}$ Psychoanalysis is predicated on the assumption that for individuals different potential worlds can be imagined and realised given self-understanding. It is to make visible and possible such alternative worlds that individuals enter psychotherapy.

This argument for necessary contingency does not depend, as in some interpretations of quantum physics, on the uncertainties imparted by the place of the observer in any process of measurement. (It is this idea which has linked the theory of relativity to the idea of 'relativism' or observationdependent understanding, in science ${ }^{8}$ ). Instead uncertainty and unpredictability is deemed to be an attribute of reality itself (Prigogine 1996, Gell-Mann 1994). Organisms, and complex organisms like human life in particular, exhibit these states of unpredictability, emergent properties, and the common 'bifurcation' of evolutionary paths, to an even greater degree than the physical universe, though within the framing of chaos theory they are

\footnotetext{
${ }^{6}$ Quinodoz (1999) writes that Ruelle, the originator of the 'strange attractor' concept, described his expression as 'psychoanalytically suggestive'. Indeed, what attractors could be stranger than those that are unconscious?

${ }^{7}$ Attempts, such as those of some forms of Marxism, to posit deterministic laws of history, have come to seem very fallible in contemporary 'post-modern' times.

8 The psychoanalytic process involves complications of this kind, since plainly the observed (the patient) is influenced by the process of observation (by the analyst) in very substantial ways. For Grunbaum $(1984,1993)$ this 'observer-effect', or the suggestibility of the patient, is the fatal flaw in psychoanalytic claims to generate knowledge. Psychoanalytic method tries to deal with this problem in various ways. Perhaps its most fundamental is the contention of object-relations theory that mental life is always organised in an ongoing relation to others, and that there could never be any way of apprehending psychic reality which did not involve a relationship with an observer. The psychoanalytic method takes specific account of this relation, via the transference and counter-transference. It thus becomes an explicit dimension of the description and explanation of the phenomena being observed, not an unnoticed distortion of what would otherwise be an 'objective' account.
} 
in this respect continuous with and not a departure from the properties of the physical world. The patterns of different evolutionary pathways, generating 'fitness landscapes' in which certain species evolve to dominate particular niches at the cost of reducing their evolutionary potential to occupy any other vacant ecological spaces, is an example of such systemic, contingent and time-irreversible patterns. (The specialisation of male peacocks in the elaboration of plumage for sexual display has for example cut off the potential of this species to evolve its capacities in other prospectively competitive attributes such as speed of flight or invisibility to predators.)

\section{Complexity Theory and Psychoanalysis}

Valuables articles by Moran (1991) Quinodoz (1997) and Miller (1999) have previously explored the relevance of chaos and complexity theory to psychoanalysis. These writers have argued that the domain of psychoanalysis is the investigation of the attributes of self-ordering multi-dimensional systems, not of the search for linear causal correlations between specified variables. There are a number of respects in which the theoretical framework generated within this paradigm provides a better fit with the models of the mind which psychoanalysis produces than theories which presuppose linear determinism. The idea of 'phase changes' between different states of equilibrium, which may be triggered unpredictably by contingencies, and which involve bifurcated developments between alternative patterns of order, meshes well with contemporary psychoanalytic theory. The concept of 'strange attractors' as the organising principles of coherent configurations of mind seems also to be a potent one, in the view of these writers.

Thus, chaos theory or complexity theory seem to offer an invaluable means of escape from the unsatisfactory choice which psychoanalysis has long seemed to face, between 'causal' and deterministic models on the one hand, derived from Freud's aspirations to scientificity, and 'hermeneutic' and 'interpretative' schemas on the other. The former recognises and seeks out relations of causality without which psychoanalysis would lack a theory of 
purposeful change or agency. But causal models of the classical kind have great difficulties in accounting for the complexity and multidimensionality of emotional and thinking processes as the essence of psychic structure and process. To be usefully applicable to mental life, the principle of causality needs to be somewhat dissociated from the idea of mechanism and determinism. Hermeneutic approaches on the other hand have the advantage that they enable analysts to follow and monitor the systems of meaning and intention, conscious and unconscious, which constitute the mental activities of their subjects. These models recognise complexity and ambiguity as essential attributes of mind, and can take account of the ways in which changes in mental process and function take place in ways which are only partially constrained by, or logically inferable from, previous structures of meaning. They allow for the emergent properties of the mind, and for the many unforeseeable connections which it makes, even if a principled disavowal of causality rules out too much. Devoted to the explication of meanings as it must be, psychoanalysis also needs a conception of the constraining powers of structures of mind, and of the causal efficacy of the analytic process in bringing about specifiable changes in the structures of the mind. It would be gravely impoverished without these.

What complexity theory suggests is that we can transcend this unwelcome dichotomy between causal reductionism on the one hand, and a merely interpretative investigation of narratives on the other. It suggests that we should be looking for order and coherence in a different place, neither in the spheres of intention and meaning alone, nor in the sphere of deterministic structures or 'mechanisms' as they have sometimes been metaphorically called. Instead, this emergent paradigm suggests, we should be looking for ordering patterns of psychic coherence, functioning within specified parameters, and for sometimes sudden changes in state, often of a binary or bifurcated kind, triggered or catalysed by external or internal factors.

Quinodoz utilises the idea of 'tuning variables' derived from chaos theory to explain how multi-dimensional systems can evolve in response to specific factors. He suggests that the intensity of a 'containing relationship' between 
infant and parents, or the intensity of a transference relationship within a personal analysis, can function as 'tuning variables' enabling development to take place along a continuum, or rather by means of step-jumps, from modes of mental functioning with fewer 'dimensions' to those with more.

British psychoanalytic thinking post-Bion has given a lot of importance to 'three dimensionality' as a criterion of psychic development. In recent work, for example by Britton, the resolution of Oedipal anxiety into the acceptance of three (or more) person relationships has been defined as a key index of psychic growth. But in reflecting on the nature of autism and post-autistic states and in seeking to understand the development of severely deprived children this tradition has also attached importance to the incorporation of the realities of time, space and causality into the understandings of the mind, as preconditions for its development. The reflexive recognition of 'inner' mental space, in the self and in others, is a third aspect of dimensionality which is now given importance in the psychoanalytic theory of development.

Whilst containing relationships can be understood as 'benign' tuning variables, we can conversely view high levels of anxiety or deprivation as 'negative' tuning variables. Where the former may support phase transitions from paranoid-schizoid to depressive modes of function (which as Quinodoz points out involves a move from fewer to more dimensions of complexity), the latter may induce shifts back towards the paranoid-schizoid end of the spectrum, with its simplified binary ordering of good and bad by mechanisms of splitting. Such high levels of psychic anxiety may be produced in populations by civil conflicts or by sudden economic insecurities, and may in their turn give rise to persecutory kinds of behaviour.

An obvious convergence between chaos and complexity theory and psychoanalysis lies in Bion's concept of catastrophic change. Within the terms of complexity theory, we can say that Bion's concept describes 'phase changes' from one state of psychic order to another potential state, providing a powerful example of the ideas of disequilibrium and changes in phasespace which achieved their broader currency through public dissemination of chaos theory and complexity considerably later. Bion's attention seems to have been drawn to Poincaré around the same time as the mathematicians 
who were developing chaos theory were beginning their work, but well before this became widely known. The interest of complexity theorists in the critical importance of the 'edge of chaos' as a state from which development and complexity arises is closely parallel to the importance and necessity of 'catastrophic change' in Bion's view of the mind. ${ }^{9}$

Recent developments in the theory of transitions and oscillations between paranoid-schizoid, borderline and depressive modes of functioning appear to be usefully framed within these notions of complex forms of order and evolution. Britton's (1998) recent theorisations of movements and oscillations between successive constellations of paranoid-schizoid and depressive functioning, each reorganising and re-framing the phenomenological contents of the temporally preceding phase in new ways, is valuable in its introduction of a temporal dimension into the idea of psychic development. One might say that the implicit aspiration of classical Kleinian theory was to devise models of the paranoid-schizoid and depressive positions which were in principle 'timereversible.' They were, that is to say, such logically consistent and closed models that it would be possible to imagine a mind ordered either by the one principle of organisation or the other, and moving backwards and forwards between them rather as one can imagine a change of physical state from liquid to gaseous and back again. What Bion's and subsequently Britton's formulations do is to add to this account a necessarily temporal dimension, acknowledging that each 'cycle' of oscillations may and indeed must be different from its predecessor in that it has to incorporate and process the psychic experiences of the previous stage. At least, in a developing mind this will happen; no doubt there are many clinical instances where there appears to be disappointingly little development, either out of the paranoid-schizoid position or even within each oscillation between paranoid-schizoid and depressive modes. But Britton's discussion of the life and work of a number of

\footnotetext{
${ }^{9}$ Another framing of these issues, termed 'catastrophe theory', arose from the work of the French mathematician René Thom (1975). This also gave rise to a research programme with many applications (Woodcock and Davis 1980, Zeeman 1977), but seems to have run out of steam . The largely U.S. based programme of development of chaos and complexity theories appears to have found greater momentum, and also a great deal of visibility through popular scientific writing.
} 
major writers (Milton, Blake, Wordsworth and Rilke) shows how this element of irreversible evolution can be seen in psychic development whatever its ultimate outcome, and can be the primary psychic subject of an artist's work. 10

Another concept in chaos theory which may have its place in psychoanalytic thinking is the idea of the 'fractal', ${ }^{11}$ which is the idea that the patterns of order which make up complex systems, bound together by 'strange attractors', may be found at all or at least many levels of a system, from the micro to the macro, and from elements of short duration to elements which persist over time. The analysis of clinical cases over time may disclose movements between paranoid-schizoid, borderline and depressive states of mind, and the triggering factors which bring such movements about. Such 'state changes' can be understood by reference to the progress or otherwise of a whole process of psychoanalytic psychotherapy, in terms of the differences between patients' states of mind at its beginning and end. . But they may also be related to the meanings of beginnings and endings for patients within more circumscribed time-intervals within a process of psychotherapy, whether these intervals be bounded by holidays, by weekend breaks, or by the beginning and end of a single session. The idea is that what is constituted by a 'paranoid-schizoid' or 'depressive' form of psychic organisation can be identified at each of these different levels and scales. It is in this sense a 'fractal', an organising pattern which manifests itself 'all the way down' within a psychoanalytic process, and in the patterns of mind of a patient which this illuminates.

Why should there be 'strange attractors', systemic forms of organisation, and irreversible evolutionary patterns, in the psychic organisations theorised by psychoanalysis? In fact, theory is psychoanalysis is largely organised around

\footnotetext{
10 There are other links to time which are important in psychotherapeutic work. One is the discovery that psychic damage and trauma can be transmitted across a generation to children, even when individuals in the parental (or grandparental) generation who were directly traumatised seem themselves to have survived. Selma Fraiberg's (Fraiberg et al 1975) concept of the 'ghost in the nursery' is one of the most influential formulations of this idea. Another, more benign instance is the "sleeper effect' described by Israel Kolvin - the evidence that long-term benefit may be obtained from psychoanalytic psychotherapy even when its short-term effects seem to have been small. (Kolvin et al 1988, Bell et al. 1989)
} 
such models. Freud's 'stage' model of oral, anal and genital development was one early version of these. The Kleinian binary model of the paranoid-schizoid and the depressive positions, is another, to which has more recently been added a third intermediary term, borderline states. Freud's classifications of neurotic pathology, Kleinian descriptions of oscillations between loving and destructive states of mind, and Rosenfeld's and Meltzer's related models of narcissistic character structure are among the many examples one could give of such systemic structures, or 'organisations', as Steiner (1993) explicitly calls them in referring to 'pathological organisations'.

Such models might have emerged merely in response to the subjective and heuristic need for coherence and simplification of complexity of practising analysts, rather than as reflections of the objective properties of their human subjects, but this seems unlikely. The reason why we posit a finite number of clearly defined 'ideal types' of psychic organisation, rather than assuming that psychic characteristics are evenly distributed along continua with no special clustering around 'extreme' or 'pure' types', is more likely to be because this is an accurate representation of psychic realities. (Gell-Mann discusses 'power laws' which map various kinds of naturally occurring distributions which suggest that regularities are a property of nature, not merely impositions by our own cognitive apparatus.) But if this is so in respect of psychic life, what are the ordering principles or 'strange attractors' which make it so?

A functional need for coherence of perception and psychic organisation, to simplify and make manageable the task of processing experience and making judgements about the world (in particular the world of other human beings) is what seems to explain the persistence of distinct patterns of psychic organisation. Symptoms, as Freud and many others have pointed out, are effective in concentrating anxiety on specific objects. They may displace anxieties from their 'real' objects, to other spheres where unconscious fears, though paralysing, are nevertheless contained by the symptom in some way. Paranoid-schizoid ways of viewing the world define the good and the bad in

\footnotetext{
${ }^{11}$ The seminal work on fractals is Benoit B. Mandelbrot The Fractal Geometry of Nature, (1977, rev. 1983). See also Stewart (1989).
} 
unambiguous terms, and eliminate confusing uncertainty and doubt. Fightflight can be a viable strategy of self-defence, which is why individuals and communities default to it when anxiety levels become high. Depressive anxieties, which involve recognition of the existence of the other, and concern for their-well-being, demand the toleration of more complex realities, and a measure of trust in the environment as not wholly retaliatory.

This 'depressive' structure requires support both from external and internal realities, and where these are lacking or weakened, it will collapse. ${ }^{12}$ We can think of the perception of external risk, and the susceptibility to internallygenerated anxieties, as different kinds of (interacting) 'strange attractors' in forming paranoid-schizoid structures of mind. It is the need to contain anxiety in some definite and unambiguous form which seems to be the common principle which determines that psychic organisations tend towards coherence, and bifurcate around different forms of coherence. Another way of putting this is to say that the coherence which analysts need to make sense of their task is also a functional need of their subjects, and indeed of all human mentality. This 'strange attractor', the principle of psychic coherence itself, is a universal, a kind of internal psychic gravitation or frictional drag, ensuring that psychic systems move in step-jumps from one form of equilibrium to another, and that their elements are rarely arrayed in random disorder. $^{13}$

The conceptions of an immanent tendency to order which are found within psychoanalysis are congruent with the more generalised conceptions of complexity and chaos theory. What is more astonishing, however, is the explicit anticipation of these ideas in the work of Bion, twenty years before the publication of most of the key modern writings in this field. In 'Learning from Experience', (1963) Bion quotes at length from Henri Poincaré (1854-1912),

\footnotetext{
${ }^{12}$ Wendy Hollway and Tony Jefferson's (2000) work on citizens' fear of crime valuably demonstrated that 'internal' realities (unconscious anxieties) were important in determining the extent of fear, independently of the external 'statistical' risks to which individuals were subject. ${ }^{13}$ Nor is random disorder as common a feature of physical nature as might be supposed. For example, pebbles on the beach, which one might think of as randomly located, are in fact sorted by the waves and tides according to their weight and volume, and are smoothed into rounded shapes by the force of friction.
} 
the mathematician who is now widely regarded as the originator of complexity theory:

$H$. Poincaré describes the process of creation of a mathematical formulation thus:

'If a new result is to have any value. It must unite elements long since known, but till then scattered and seemingly foreign to each other, and suddenly introduce order where the appearance of disorder reigned. Then it enables us to see at a glance each of these elements in the place it occupies as a whole. Not only is the new fact valuable on its own account, but it alone gives value to the old facts it unites. Our mind is as frail as our senses are; it would lose itself in the complexity of the world if that complexity were not harmonious; like the short-sighted, it would only see the details, and would be obliged to forget each of these details before examining the next, because it would be incapable of taking in the whole. The only facts worthy of our attention are those which introduce order into this complexity and so make it accessible to us. 14

Bion derives his concept of the 'selected fact' from Poincaré's insight. Bion put it thus:

I have used the term 'selected fact' to describe that which the psycho-analyst must experience in the process of synthesis. The name of one element is used to particularise the selected fact, that is to say the name of that element in the realisation which appears to link together elements not hitherto seen to be connected... The selected fact is the name of an emotional experience, the emotional experience of a sense of discovery of coherence.

Learning from Experience (chapter 23, p. 72)

Bion is here arguing for the primacy of experience of psychic reality over deductive law-like formulations, arguing that the latter can only be made once links have been made in the mind ('epistemological' links) between experienced phenomena. He suggests that Poincaré's description of finding

${ }^{14}$ H. Poincaré. (1952) Science and Method. (Originally published in France in 1908). 
harmonious order in complexity 'closely resembles the psycho-analytical theory of paranoid-schizoid and depressive positions adumbrated by Mrs Klein.'

This text of Bion was of course central to his own reorientation of psychoanalysis towards the primacy within it of imaginative experience, which he said called for 'negative capability' (Keats) as the precondition of understanding. Meltzer's subsequent characterisations of the aesthetic aspects of mental function developed from this idea. Bion of course also remained committed to the relevance of logical deductive systems and categorisations. However he thought that these followed from a prior 'emotional experience' of finding coherence in disordered and fragmented states, and could not precede it. His concept of catastrophic change, as we have said, directly mirrors the concepts of changes of state which are elaborated within chaos theory, and are there given mathematical formulations which seem unlikely to be achievable within the 'open systems' of the mind.

It is noteworthy that the mathematical aspirations of Bion's work which probably aroused most scepticism among many psychoanalysts, and have been most difficult for them to follow up, were in fact responsible for his anticipation of this new and valuable framing of scientific understanding. Psychoanalysis has been sustained throughout by such links (sometimes like this one unexpected ones) with different fields of inquiry. Indeed such conjunctions may embody the kind of creative catastrophic change which Bion's psychoanalytic theory predicts and prescribes as essential to development.

Bion's concept of 'selected fact' was later taken up by Edna O'Shaugnessy and other analysts ${ }^{15}$ in the elaboration of the idea of the specifically 'clinical

\footnotetext{
${ }^{15}$ See the special $75^{\text {th }}$ Anniversary Issue (Vol. 75, 1994) of the International Journal of Psychoanalysis on 'The Conceptualisation and Communication of Clinical Facts in Psychoanalysis.'
} 
fact' of the psychoanalytic session. This, in O'Shaugnessy's paper (1994), refers to a perception of the reality of the transference relationship at a given moment which can be successfully communicated to the patient. When a clinical fact has been correctly apprehended, It is not only the analyst, but also the analysand, who has this experience of perceiving an unexpected coherence and meaning. This possibility of shared understanding is of course essential to the psychoanalytic process, and develops Strachey's (1934) earlier idea of 'mutative interpretation'. This is one aspect of the way in which Bion's early anticipation of the insights of complexity theory has informed contemporary psychoanalytic thinking. ${ }^{16}$

The Relevance of Complexity Theory to Infant Observation.

What the above argument suggests is that researchers in psychoanalytic infant observation should be looking primarily not for causal correlations or sequences of linear development, but for ordering patterns, for the evidence of emergent systemic organisation in the minds of infants and in the relationships between infants and those around them. There may be a good fit between the frame of inquiry set out by complexity theory, and the procedures and techniques of 'naturalistic' infant observation, just as there has been shown to be such a fit with the procedures of clinical research. What characterises infant observation is a holistic approach, an openmindedness in regard to internal and external aspects of the experience of infants and their families, and an interest in mapping changes and development over time, which is expressed in its predominantly narrative, case-study approach. What may be captured through these methods is a recognition of multi-dimensional patterns of organisation (for example, the effects of a supportive or non-supportive presence of grandparents, or of a

\footnotetext{
${ }^{16}$ There is a reflexive aspect to the apparent correspondence between the theoretical selforganising systems posited in modern psychoanalytic theory (the 'pathological organisations' of Steiner, for example) and complexity theory. On the one hand, psychic structures do have this character. On the other hand, those psychic structures which have been categorised and defined in post-Kleinian psychoanalysis in particular have been deeply influenced by a psychoanalyst, namely Bion, who grasped the essence of chaos and complexity before this paradigm acquired a name, and have thus already been shaped by this way of thinking.
} 
persecuting or benign internal mother); the identification of discrete 'changes of state' or shifts of equilibria, sometimes through an observed crisis in relationships or in the observational setting; or the role of 'tuning variables' of a benign form of containment, or its absence, in the psychic development of the infant. Researchers in infant observation need to clarify and systematise both the 'structures' and 'patterns' which can be established as norms for their observations, and the observable 'indicators' which tell them about the existence or strength of such a pattern.

Much of the literature on psychoanalytic infant observation describes exactly these forms of discovery. One of the innate principles of complexity theory, namely the idea that there is an inherent tendency to order in apparently random phenomena, is fundamental to Bick's approach to the mind of the infant. She posited a need for the binding together of the self and its bodily and mental experiences as a primordial psychic fact. The principal function of maternal containment is to support this sense of coherence, and to protect the infant against anxieties of 'falling to pieces'. Bick's most important substantive discovery, her theory of the 'Second Skin' (1968), has this primordial anxiety as its backdrop:

The thesis is that in its most primitive form the parts of the personality are felt to have no binding force amongst themselves and must therefore be held together in such a way that it is experienced by them passively, by the skin functioning as a boundary. But this internal function of containing the parts of the self depends initially on the introjection of an external object, capable of fulfilling this function. Later, identification with this function of the object supersedes the unintegrated state, and gives rise to the fantasy of internal and external spaces.

Bick went on to describe how

The need for a containing object would seem, in the infantile unintegrated state, to produce a frantic search for an object - a light, a voice, a smell, or 
other sensual object - which can hold the attention and thereby be experienced, momentarily at least, as holding the parts of the personality together. The optimal object is the nipple in the mouth, together with the holding and talking and familiar smelling mother. Material will show how this containing object is experienced concretely as a skin. Faculty development of the primal skin function can be seen to result either from defects in the adequacy of the actual object, or from fantasy attacks on it, which impair introjection. Disturbance in the primal skin function can lead to the development of a 'second skin' formation through which dependence on the object is replaced by a pseudo-independence, by the inappropriate use of certain mental functions, or perhaps innate talents, for the purpose of creating a substitute for this skin container function.'

In one of the seminal psychoanalytic contributions to the infant's psychic development, she defined this, drawing on infant observational case examples, as the 'second skin' formation. (Bick 1968).

In her essay Notes on Infant Observation in Psychoanalytic Training (1964) Bick makes frequent to the 'patterns of behaviour' which are discerned by observers in infants. She describes these patterns in very concrete terms for example, the different gestures of a baby's hands in relation to its mother's two breasts - and offers many conjectures on what these may signify in terms of psychic development. For example in that case these different gestures suggest a kind of incipient splitting. The idea that 'patterns' are what one should be looking for, whether in the minutiae of physical movements, or in a broader mind-body configuration such as that of the 'Second Skin', is an example of the kind of structure which one might expect to find in open self-organising systems, especially but not exclusively in living forms, and in human subjects in particular. ${ }^{17}$

\footnotetext{
${ }^{17}$ Once again, the convergence of Bick's ideas with the underlying conceptions of complexity theory, and with the ideas of Bion which anticipate these, was only to be expected. Bick refers
} 
Many of the most fertile insights of infant observation research to date are of this kind. To the example of Bick's papers, one could add many others, including Juliet Hopkins' (1996) article on the pattern and consequences for development of 'too-good mothering,' and the work of Sue Reid (1997) on observed patterns of mother-infant interaction that seem conducive to the development of autism. Another recent example of the identification of a 'pattern' of this kind is in Pamela Sorensen's (2000) paper 'Observations on transition facilitating behaviour - developmental and theoretical implications'. This paper, which seeks to establish a bridge between attachment and object relations theories, clarifies the importance of everyday transitions for all babies, from observations of the experiences of exceptionally vulnerable infants in a Neo-Natal Intensive Care Unit. The exploration and elaboration of different forms of containment has become one of the principal areas of infant observational research (Briggs 1997). Along with a developing capacity to discriminate different patterns of containment, including those involving severe deficits or disturbances, have come the beginnings of purposefully 'therapeutic' infant observations, where some measure of deliberate intervention takes place. This reflects a greater confidence in the ability of observers to understand from an early stage what is going on in an infant's relations to those close to him. But as in psychotherapy itself, the possibility of testing the outcome of interventions is likely to provide an additional resource for the development of new understandings and concepts.

Once theoretical classifications have become established as stable points of reference, they become capable of further differentiation, even 'bifurcation' to use the terminology of complexity theory, (as in the differentiation by Rosenfeld between libidinal and destructive narcissism). It is in this way that the adequacy and complexity of the theoretical models available for researchers can develop as they conduct observations.

The assumption of complexity theory that realities are complex, emergent, non-reversible over time, and liable to generate increased difference, is consistent with both the scientific and humanistic assumptions of

to 'situations conducive to catastrophic anxieties in the unintegrated state' in her Second Skin paper of 1968, indicating her closeness to Bion's thinking at that time. 
psychoanalysis. Humanistic assumptions because psychoanalysis is committed to the inherent value of autonomy, difference, and choice - it wants its subjects to be understood and to understand themselves in their particularity, not merely as an instance of a diagnostic category. And scientific because the theoretical models of psychoanalysis have never been intended to do more than define the boundary conditions of different kind of mental and emotional life, within and between which much variation is both anticipated and desired.

The specific focus of psychoanalytic infant observation, conducted in natural settings, contrasts with the investigations of infant development undertaken within the 'attachment theory' perspective, though their findings are in some respects mutually supportive. Attachment theory is wedded to a more classical scientific model, being committed to the identification of definite causal patterns or models of attachment and its preconditions. These models do capture some important boundary conditions which determine the capacity for relationships and well-being. In doing so, they identify temporal and relational spaces where developmental vulnerability lies, and in which preventive interventions are feasible and desirable. But these approaches seem to have stopped short of much further differentiation, once they had established their basic array of framing conditions. (Three main kinds of attachment - securely-attached, anxious attached avoidant, anxious attached ambivalent/resistant, were discovered by Mary Ainsworth (Ainsworth et al. 1978), and a fourth - the disorganised/disoriented was subsequently and valuably added by Mary Main. (Main and Solomon 1986, Main and Hesse 1990). This experimental methodology seems to have only limited conceptual resources with which to map differences between individuals, or their patterns of psychological development. ${ }^{18}$ We could say, following GellMann's term for broader interpretative schema, that these models are 'coarse-grained' simplifications of realities which psychoanalysis and psychoanalytic infant observation seek to investigate in more 'fine-grained'

\footnotetext{
${ }^{18}$ Peter Fonagy's comments on the challenges posed by psychoanalysis to attachment theory in chapter 13 of his Attachment Theory and Psychoanalysis (2000) are perceptive about their differences of approach.
} 
ways. The finer the grain of observation, the more that contingencies and differences become evide

t.

The increasing emphasis within the attachment theory tradition on 'mental models and maps' has increased the explanatory coherence of its models, and brought it closer to psychoanalysis, in part through the incorporation of some of Bion's ideas. We could say that the capacity for the mental processing of experience is functioning within this paradigm as the 'strange attractor' which hold its different patterns of attachment together. But it continues to lack the multi-dimensionality of the psychoanalytic theory of personality development. Psychoanalysis, it will be remembered, takes account of the dimensions contributed by predispositions to love and hate, as well as by the capacity for understanding, and by the role of unconscious representations and memories internalised as phantasies of self and others. It seeks to investigate in its fine-grained way the idiosyncratic 'scripts', both conscious and unconscious, which evolve as means of dealing with emotionally-charged realities.

There is a natural continuum and complementarity between fine- and coarsegrained forms of explanation, and between the more conjectural and complex forms of explanation which go with the first, and the more definite and categorical causal explanations which go with the second. One can say therefore that both clinical and observational psychoanalytic approaches, and protocol-driven and laboratory-based attachment theory procedures, have their necessary and distinct place in the investigation of infant development. (Rustin 2001a).

What about the role of causality within these different models? Should psychoanalysis, and infant observation research in particular, be seeking to establish causal laws, or not? In what way, if any, does the contribution of complexity theory resolve the antithesis between 'interpretative' and 'causal' 
models of explanation which we posited at the outset as a long-standing dilemma for psychoanalysis?

The identification of 'patterns', 'self-organising systems', and 'strange attractors' within complexity theory substitutes complex and holistic notions of causal relation for the 'linear' models aspired to by many mechanistic sciences, including much of psychology. Complexity theory, and its applications within psychoanalysis, identifies 'fields of force' which bind psychic phenomena, and which create or constitute dispositions for subjects to act according to discernible patterns. The paranoid-schizoid and depressive positions are examples of dispositions of this kind. These are 'generative structures', to use another terminology, ${ }^{19}$ with many connected dimensions. Their value for psychoanalysts, whether as clinicians or researchers, is not as predictors of specific acts, but as binding conceptions which specify tendencies or dispositions with many connected dimensions, for example of thought, feeling, and behaviour. It is possible to identify typical causal connections within these theoretical schema, and to find ways of accurately measuring the incidence of these. (Bick for example suggested that certain kinds of deficient containment would lead to defences of pseudoindependent 'muscularity'; there is no reason to think that this could not be treated as a definite hypothesis and specifically tested against evidence.) But the interest and value of these models lies in their capacity to give definition to many differentiated kinds of pattern, as these appear in observational or clinical settings. This is why its preferred style of thinking is in terms of multidimensional narratives, rather than by the correlation of discrete variables. These models are not well adapted to demonstrating specific linear causal

\footnotetext{
19 The concept of 'generative structures' derives from Roy Bhaskar's realist theory of science (Bhaskar 1975). According this view, theories identify and model structures which are manifested primarily through their observable effects. Scientific inquiry therefore involves not only making empirical observations, and finding correlations between them, but also making inferences from observed data to underlying structures and mechanisms. Reviews of this approach in relation to psychoanalysis are in Will $(1980,1986)$ and Rustin (1991b). Psychoanalysis is distinctive in its assumption that surface manifestations of consciousness and behaviour are derived causally from structures of mind which are held to be effective at 'deep' levels of the mind, and whose existence and power has to be inferred from the 'surface' phenomena of consciousness. Freud's theory of repression explained why these structures remained largely 'unconscious' . The idea of 'mechanism' becomes important in
} 
effects between identified variables, such as evidence-based policy might wish to see. But they are powerful in identifying more holistic patterns of behaviour, and their antecedents and effects, and enabling skilled therapists to identify within these patterns areas in which change and development is possible. $^{20}$

Psychoanalysis is antithetical in this respect to attachment theory, which gives much higher priority to the simplifications and generalisations necessary to establish robust causal connections (which of course it has done with success). The value of complexity theory for psychoanalysis is that it provides a much more adequate meta-framework for its ways of thinking than the mechanistic models that have earlier dominated the sciences. There is a large domain of nature, it suggests, that is neither determined in the manner of a closed mechanism or system, nor wholly random, indeterminate, or 'free'. Instead, it posits self-organising systems, of high complexity, and indeterminacy within understood limits. Precisely, in fact, the world of experience with which clinical psychoanalysts continually struggle, and which observers encounter on a weekly basis in their visits to infants and their families.

In the last forty years, since the publication of T.S. Kuhn's Structure of Scientific Revolutions, (1962) the recognition of the actual diversity and complexity of the sciences has made it easier for psychoanalysts to locate their work as having a place within a larger scientific community, its methods seeming less anomalous once they are understood as appropriate to their

psychoanalytic discourse through Anna Freud's theory (A. Freud 1936) of mechanisms of defence.

${ }^{20}$ Another model of explanation which is relevant here is the 'part-whole' analysis which is advocated by Thomas Scheff (1997) as often most appropriate in the human sciences. Where self-maintaining systems exist - for example in biological organisms, or in social organisations or processes - the most useful form of explanation may be to clarify the relation of specific phenomena to the larger structure and process of which they form part.

'Catastrophic changes' from one pattern of systemic coherence to another create new partwhole relations, which then become a matter for investigation. Psychoanalytic interpretation often looks for connections of this kind. On the part-whole analysis of narrative texts, see Wengraf (2001)). 
specific topics of study. ${ }^{21}$ It seems likely that 'Complexity Theory' offers a further illumination of this kind. It turns out that complexity, emergent properties, susceptibility to phase-transitions ('catastrophic change', in Bion's terms, (1965, 1970), individual difference, and ubiquitous contingency, may be normal facts of mental life, not merely the imprecise reflections of the inadequate scientific method of psychoanalysis. Not only are these complex and seemingly chaotic structures characteristic of the psychic realities which psychoanalysis investigates, but they seem now to have been found to be the attributes of a good part of biological and material nature besides.

-end-

\section{References}

Ainsworth, M.D.S., Blehar, M.C., Waters, E. and Wall, S. (1978) Patterns of Attachment: a Psychological Study of the Strange Situation. Hillsdale, N.J.: Erlsbaum.

Bell, V., Lyne S., Kolvin, I.(1989) 'Play Group Therapy: Processes, Patterns and Delayed Effects', in Schmidt, M.H. and Remschidt, H. (eds) Needs and Prospects of Child and Adolescent Psychiatry. Stuttgart: Hogrefe and Huber. Bhaskar, R. (1975). A Realist Theory of Science. Leeds: Leeds Books.

Bick, E. (1964) 'Notes on Infant Observation in Psycho-analytic Training,' International Journal of Psychoanalysis, Vol. 5. (repr. in W.H. Williams (ed.)

${ }^{21}$ On the diversity of methods even in the natural sciences, see Galison and Stump (1996) 
(1987) Collected Papers of Martha Harris and Esther Bick. Perthshire: Clunie Press.

Bick, E. (1968),'The Experience of the Skin in Early Object Relations,' in International Journal of Psychoanalysis, Vol. 49, P. 484. (repr. in Williams 1987) and Spillius, 1988).

Bion, W.R.(1963) Learning from Experience. London: Heinemann. (repr. H. Karnac 1984).

Bion, W.R. (1965) Transformations. London: Heinemann. (repr. H. Karnac 1984).

Bion, W.R. (1970) Attention and Interpretation. London: Heinemann. (repr. H. Karnac 1984).

Briggs, S. (1997) Growth and Risk in Infancy. London: Jessica Kingsley.

Britton, R. (1998) Belief and Imagination: Explorations in Psychoanalysis. London: Routledge/Institute of Psychoanalysis.

Byrne, D. (1998) Complexity Theory and the Social Sciences: an Introduction. London: Routledge.

Eve, R.A., Horsfall, S. and Lee, M.E. (eds) (1997) Chaos, Complexity and Sociology. London: Sage.

Fraiberg, S.H., Adelson, E. \& Shapiro, V. (1975) 'Ghosts in the Nursery: A psychoanalytic approach to the problems of impaired infant-mother relationships.' Journal of the American Academy of Child Psychology, 14, 387-422.

Freud, A. (1936) The Ego and the Mechanisms of Defence. London: Karnac (revised 1993).

Galison, P. and Stump D.J. (eds) (1996) The Disunity of Science: Boundaries, Contexts and Powers. Stanford Ca. Stanford University Press. Geertz., C. (1973) The Interpretation of Cultures. New York: Basic Books. 
Gell-Mann, Murray (1994) The Quark and the Jaguar: Adventures in the Simple and the Complex. London: Abacus.

Gleick, J. (1998) Chaos and the Science of the Unpredictable. London: Vintage.

Grunbaum, A. (1984) The Foundations of Psychoanalysis: a Philosophical Critique Los Angeles: University of California Press.

Grunbaum, A. (1993) Validation in the Clinical Theory of Psychoanalysis. Madison CT: International Universities Press.

Hollway, W., and Jefferson, T. (2000) Doing Qualitative Research Differently: free association, narrative and the interview method. London: Sage.

Hopkins, J. (1996) 'The dangers and deprivations of too-good mothering.' Journal of Child Psychotherapy. Vol. 23. 2, pp 407-422.

Kauffman, S. (1995) At Home in the Universe. London: Viking.

Knorr-Cetina, K. (1999) Epistemic Cultures: How the Sciences make Knowledge. Cambridge Ma: Harvard University Press.

Kolvin, I., Macmillan A., Nicol, A.R. Wrate, R.M. (1988) 'Psychotherapy is effective', Journal of the Royal Society of Medicine, Vol. 81, No, 5 261-266. Kuhn, T.S. (1962) The Structure of Scientific Revolutions. Chicago: Chicago University Press.

Likierman, M. (1989) 'The Clinical Significance of Aesthetic Experience.' International Review of Psychoanalysis, vol. 16, part 2.

Main, M. and Solomon J. (1986) "Discovery of an insecure/disorganised/disoriented attachment pattern. In Affective Development in Infancy, ed. T. B. Brazelton and M.W. Yogman, pp. 95-125. Norwood, N.J.: Ablex.

Main M. and Hesse E. (1990) 'Parents' unresolved traumatic experiences are related to infant disorganised attachment status: is freightened and/or frightening parental behaviour the linking mevchanism?' in Attachment in the Pre-School Years: Theory, Research and Intervention, ed. M Greenberg, D. 
Cicchetti and E.M. Cummings pp. 161-182. Chicago: Chicago University Press.

Mandelbrot, B.B. (1983) The Fractal Geometry of Nature (rev.ed). New York: W.H. Freeman.

Miller, M.L. (1999) 'Chaos, Complexity and Psychoanalysis.' Psychoanalytic Psychology. Vol 16, No. 3.

Moran, M.H. (1991) 'Chaos theory and psychoanalysis: the fluidistic nature of the mind.' International Review of Psychoanalysis Vol. 18, No. 2 Pp. 211221.

O'Shaugnessy, E. (1994) 'What is a clinical fact?' International Journal of Psychoanalysis Vol 75, pp 939 -947.

Poincaré, J.H. (1908/1952) Science and Method. New York: Dover.

Prigogine I., and Stengers, I. (1984) Order out of Chaos. New York: Bantam.

Prigogine, I. (1996) The End of Certainty. New York: Free Press.

Quinodoz, J.-M. (1997) 'Transitions in psychic structures in the light of deterministic chaos theory.' International Journal of Psychoanalysis Vol. 78, No. 4. Pp 699-718.

Reid, S., (1997) 'Projective Identification: the Other Side of the Equation', Part 2 of 'The Generation of Psychoanalytic Knowledge: Sociological and Clinical Perspectives, British Journal of Psychotherapy, Vol. 13, No 4,. 542 554.

Robinson, S. (2002) 'What gets measured, gets delivered? ' Psychoanalytic Psychotherapy, Vol. 16, 1, pp 37-57.

Ruelle, D. (1991) Chance and Chaos. Harmondsworth: Penguin

Rustin, M.J. (1989) 'Observing Infants: Reflections on Methods,' in L. Miller, M.E. Rustin, M.J., Rustin, J. Shuttleworth (eds). Closely Observed Infants. London: Duckworth.

Rustin, M.J. (1991a) The Good Society and the Inner World. London: Verso. 
Rustin, M.J. (1991b) 'Psychoanalysis, Philosophical Realism, and the new New Sociology of Science', in Rustin (1991a).

Rustin M.J. (1991c) 'Psychoanalysis and Aesthetic Experience', in Rustin (1991a).

Rustin, M.J. (1997b) 'What do we see in the Nursery? (International Journal of Infant Observation, Vol. 1, No.1. (repr. in Rustin 2001a)

Rustin, M.J. (2001a) Reason and Unreason: Psychoanalysis, Science and Politics. London:Continuum.

Rustin, M.J. (2001b) 'Research, evidence and psychotherapy', in Mace, C., Moorey, S. and Roberts, B. Evidence in the Balance: a Critical Guide for Practitioners. Hove: Brunner-Routledge. (repr. in Rustin 2001a)

Scheff, T. (1997) Emotions, the social bond and human reality; part/whole analysis. Cambridge: Cambridge University Press.

Sorensen, P. B. (2000) 'Observations of transition-facilitating behaviour developmental and theoretical implications', International Journal of Infant Observation', Vol.3, No.2.

Spillius, E. (1988). Melanie Klein Today: Vol. 1: Mainly Theory. Vol. 2: Mainly Practice. London: Routledge.

Steiner, J. (1993) Psychic Retreats. London: Routledge

Stewart, I. (1990) Does God Play Dice? The New Mathematics of Chaos. . London: Penguin.

Strachey, J. (1934) 'The nature of the therapeutic action of psychoanalysis,' in International Journal of Psychoanalysis, Vol. 15, pp. 127 - 159.

Taylor, M.C. (2001) The Moment of Complexity: Emerging Network Culture. Chicago: Chicago University Press.

Thom, R. (1975) Structural Stability and Morphogenesis: an Outline of a General Theory of Models. Reading: Benjamin.

Thrift, N. (1999) 'The Place of Complexity', Theory, Culture and Society. No 3, pp. 31-69. 
Waldrop, M.M. (1992) Complexity: The Emerging Science at the Edge of Order and Chaos. New York: Simon and Schuster.

Wengraf, T. (2001) 'Uncovering the general from within the particular: from contingencies to typologies in the understanding of cases,' in P.M.

Chamberlayne, J. Bornat and T. Wengraf. (eds.) The Turn to Biographical Methods in Social Science. London: Taylor and Francis/Routledge.

Will, D. (1980). 'Psychoanalysis as a Human Science,' British Journal of Medical Psychology 53: 201-211.

Will, d. (1986) 'Psychoanalysis and the New Philosophy of Science.'

International Review of Psychoanalysis 13: 163-173.

Woodcock, A. and Davis, M. (1980) Catastrophe Theory. Harmondsworth: Penguin.

Zeeman, C. (1977) Catastrophe Theory: Selected Papers 1972-1977.

Reading: Benjamin. 